\title{
REVIEW
}

\section{The effectiveness of magnetic resonance imaging-guided focused ultrasound thalamotomy in the treatment of patients with essential tremor: a literature review}

\author{
Alain Nathan Sahin $\mathrm{MSc}^{1}$ \\ ${ }^{\prime}$ Class of 2020, Faculty of Medicine, Université de Montréal
}

\begin{abstract}
Purpose: Essential tremor (ET) may eventually become resistant to drugs, and invasive surgeries may have side effects. Magnetic Resonance Imaging-guided focused ultrasound (MRIgFUS) thalamotomy is being tested as an alternative treatment. This literature review sought to explore the effectiveness of the use of transcranial MRIgFUS thalamotomy on patients with ET.
\end{abstract}

Methods: To identify potentially relevant original English articles, PubMed and Google Scholar were searched until January 1, 2016. Title and abstracts and then full texts, were screened for inclusion criteria. Articles discussing the effectiveness of the transcranial MRIgFUS thalamotomy for the treatment of ET in humans were included. Methods, results and discussion sections of selected articles were synthesized.

Results: Four articles were included in the synthesis. Overall, 37 patients with ET were treated with transcranial MRIgFUS thalamotomy. Studies found that scores for hand tremor, disability, and quality of life significantly improved. Treatment had some temporary side effects such as sensory, cerebellar, motor, speech abnormalities; however, paresthesias persisted in some patients. Monitoring potential serious side effects such as intracerebral hemorrhage and neurologic impairment was recommended since these were previously found to be associated with other types of surgical thalamotomy procedures.

Conclusions: Although most of the studies were uncontrolled and potential side effects need to be considered, results of transcranial MRIgFUS thalamotomy for ET in humans suggest that this treatment has potential to improve patients' quality of life. Randomized trials with larger sample sizes are warranted, and one of these is underway.

$\mathrm{T}$ he most common adult-onset movement disorder is essential tremor (ET). The prevalence of ET is $0.9 \%$ in the general population, increases with age (4.6\% in those aged 65 and over), and peaks in early adulthood or at the age of $60 .^{1,2}$ Although the cause of ET is still unclear, a genetic component is important. ${ }^{3}$ ET is characterized by uncontrollable shaking, or tremors, in different body parts and on different sides of the body. Eighty five percent of ET patients report that this condition affects their quality of life, and 15\% report that it causes significant disability. ${ }^{2}$

There are medications to treat the trembling in hands, head, or voice seen in this condition, yet 30\% of patients are resistant to pharmacotherapy. ${ }^{2}$ In these cases, various surgical treatment strategies may be considered. For instance, the ventral intermediate nucleus of the thalamus can be targeted by radiofrequency identification, high-power X-rays, or gamma radiation. Another surgical alternative is deep brain stimulation. In this procedure, an electrode is implanted in the brain and blocks abnormal brain signals causing tremor. However, all these techniques are invasive, expose patients to ionizing radiation, and may have side effects such as infection, hemorrhage and brain tissue damage. $^{2}$
Magnetic resonance imaging (MRI) guided focused ultrasound thalamototy (MRIgFUS) has been introduced as a promising alternative and is being tested as a new treatment for ET. ${ }^{4}$ MRIgFUS is considered an important advancement in the non-invasive treatment of neurologic disorders. First, it minimizes the occurrence of the above-mentioned complications by ablating tissues non-invasively. Second, MRI technique precisely guides transcranial sonication to the treatment location, and thermal imagery allows a real-time monitoring of the sonication intensity. ${ }^{3}$

To date, there is no comprehensive review of the results of this novel treatment in ET. This literature review sought to explore the effectiveness of the use of transcranial MRIgFUS thalamotomy for the treatment of patients with ET. Specific objectives of the review were 1) to find original studies reporting on the results of MRIgFUS for ET in humans and 2) to describe the effectiveness (either success or failure) and the side effects of the treatment.

\section{Methods}

To identify potentially relevant English journal articles, PubMed and Google Scholar were searched until 
January 1, 2016 without time limitation. The following specific key words or phrases were used to identify the relevant articles: "magnetic resonance imaging", "MRI-guided", "Image-guided", "essential tremor", "thalamotomy", "thermal ablation", neurosurgery", and "focused ultrasound". Duplicates were removed among retrieved articles. Titles and abstracts met inclusion criteria if they were original studies conducted in human subjects, used MRIgFUS, and thalamotomy was performed for treatment of ET. Full texts of the remaining articles were reviewed and the same inclusion criteria were applied. The methods, results, and discussion sections of these selected articles were synthesized.

\section{Results}

Figure 1 presents the results of the search. The titles and abstracts of 306 potentially relevant documents were screened for the inclusion criteria. Of those, 297 were excluded for the following reasons: 193 were review, commentary, editorial, abstract, or technical papers; 22 did not include MRIgFUS; 55 studied conditions other than ET; and 27 were not conducted in humans. Nine full texts were examined in detail for inclusion. Of those, four articles were included in the final synthesis. Studies were conducted in the USA, Canada, and Korea. Characteristics and findings of those selected articles are shown in Table 1. Table 2 outlines inclusion criteria amongst three studies.

Elias et al. conducted an uncontrolled pilot study. ${ }^{5}$ Fifteen patients with severe, medication-refractory ET, who had not undergone previous stereotactic or cranial surgeries and whose dominant hand was the most severely affected extremity, were enrolled. Scores for hand tremor, disability scores, and quality-of-life scores significantly improved with MRIgFUS. ${ }^{5}$ However, in this sample of 15 patients, transcranial MRIgFUS thalamotomy had some side effects such as transient sensory, cerebellar, motor, and speech abnormalities, and four patients had persistent paresthesias. Since other types of surgeries, such as radiofrequency thalamotomy and deep-brain stimulation, have been found to be associated with potential serious side effects (intracerebral hemorrhage and neurologic impairment including hemiparesis, ataxia, and dysarthria) the authors suggest keeping in mind the possibility of the occurrence of these events after performing MRIgFUS thalamotomy.

Wintermark et al. published the results of further analyses of the same sample (the pilot study conducted by Elias et al.) in two articles. ${ }^{6,7}$ Although these articles were not included in the review sample, it is worthwhile to discuss their results in this review. First,

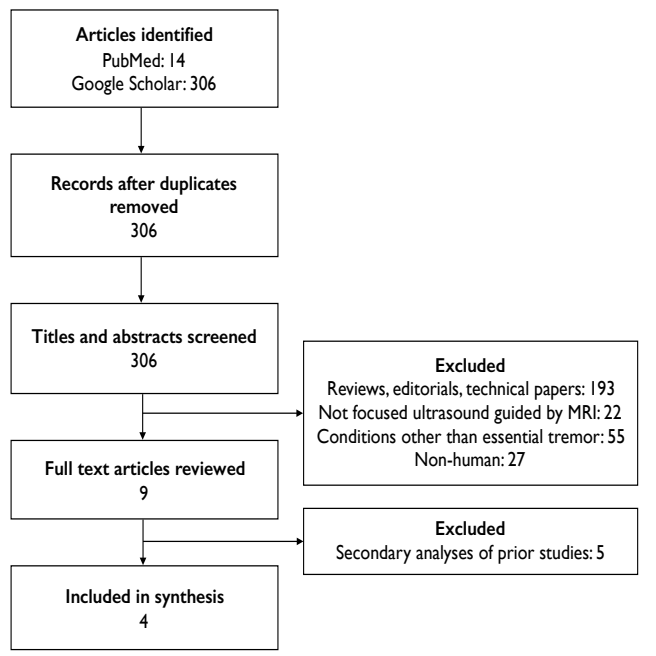

Figure 1. Flow chart of search results.

they investigated the relationship between the total lesion size after treatment and the clinical response to the MRIgFUS treatment based on the Clinical Rating Scale for Tremor (CRST) score. They found that tremor control was maximal at one week when the lesion size was larger and decreased at one or three months when the perilesional edema had resolved and the total lesion size was smaller. ${ }^{6}$ Second, they used diffusion MRI technique and fractional anisotropy (FA) as an imaging feature signifying tissue disruption or disorganization. ${ }^{7} \mathrm{FA}$ is a relative quantity describing the variance between the levels of water molecule diffusion in different directions to reveal microscopic details about tissue architecture, either normal or in a diseased state. ${ }^{8}$ Wintermark et al. found a strong correlation between a decrease in FA and clinical improvement in hand tremor three months after lesion induction. ${ }^{7}$

Lipsman et al. observed instantaneous and persistent improvements in tremor in the dominant hand of four patients treated with transcranial MRIgFUS thalamotomy in an uncontrolled study. ${ }^{9}$ They found that the patients had functional benefits, showing improvements in writing and motor tasks. They too suggest monitoring potential progressive neurological deficits after the procedure. ${ }^{9}$

Jung et al. treated eleven patients with drug refractory ET referred from movement neurologists or psychiatrists (no other eligibility criteria were reported). They observed that the lesions were able to be seen immediately after treatment and noticeably reduced in size over time. ${ }^{10}$ However, in three ET patients, there was no or little temperature rise (i.e., $<52^{\circ} \mathrm{C}$ ) that would be enough to cause lesions in thalamus during MRIgFUS. They observed differences in their ratio of cortical-to-bone marrow thickness (i.e., skull 


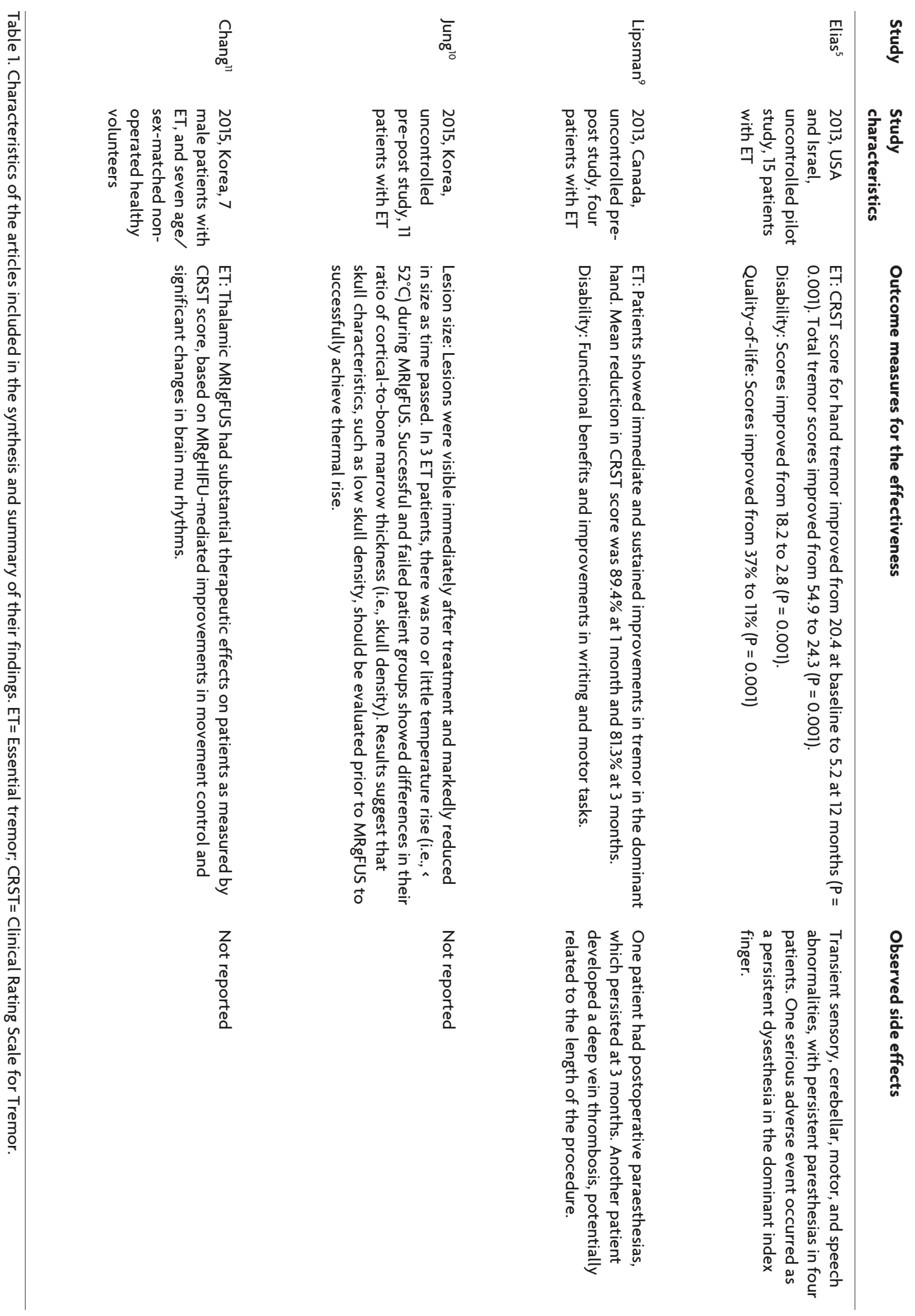


Eligibility criteria

Aged between 18 and 80 years
Elias et al.

$N / M$

Sample age mean (SD)

is $66.6 \pm 8.0$ years

(range, 53 to 79 )

Able and willing to give consent and to attend all study visits

ET diagnosis confirmed by a neurologist specializing in movement disorders

Able to communicate sensations during treatment

Clinically significant tremor defined as a score of 2 or more on the postural or action item on the Clinical Rating Scale for Tremor (ranging from 0 to 4) in the dominant hand

Tremor refractory to adequate trials of at least two medications, one of which should be either propranolol or primidone

Stable doses of all medications for 30 days prior to study entry and for the duration of the study

The ventralis intermedius region of the thalamus must be apparent on MRI such that targeting can be done with either direct visualization or by measurement from known anatomic landmarks

Not having history and/or presence of psychiatric disease, previous and/or evidence of substance abuse

Not having presence of significant cognitive impairment as determined by a score of 24 or less on the MiniMental State Examination

Not having neurodegenerative conditions (e.g., Parkinson's disease, Alzheimer's)

Not having presence of deep-vein thrombosis (by ultrasonography of the legs), unstable cardiac conditions, or a coagulopathy

Not having undergone previous stereotactic or cranial surgery

Not having standard contraindications to MRI and known intolerance or allergies to the MRI contrast agent

Other ruled-out conditions

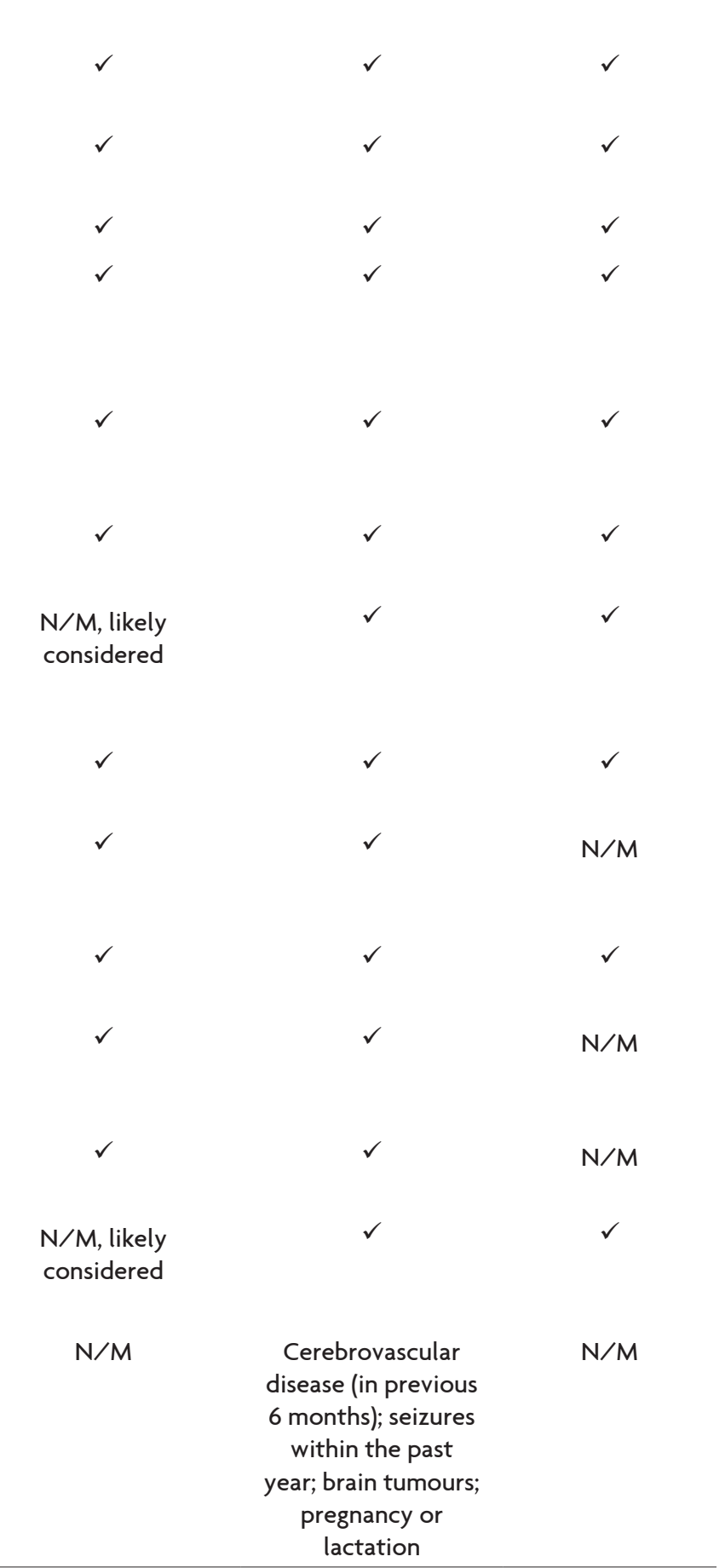

Lipsman et al. Chang et al.

)

$\checkmark$


density) between successful and failed patient groups. Therefore the authors suggest that skull characteristics, such as low skull density, should be evaluated prior to MRIgFUS in order to successfully achieve the thermal rise required for the ablation of the targeted area.

From a different perspective, Chang et al. compared the effects of the treatment in seven male patients and seven age/sex-matched non-operated healthy volunteers and found significant changes in brain rhythms. ${ }^{11}$ They used CRST for patients with ET as a primary outcome measure and also compared the MRIgFUS-mediated reduction in magnetoencephalography correlates of patients with ET to the level observed in age/sex-matched non-operated healthy subjects.

\section{Discussion}

This review provides an exploratory synthesis of the current knowledge about potential effectiveness of the transcranial MRIgFUS thalamotomy for the treatment of ET in humans. Overall, 37 patients with ET have been treated with transcranial MRIgFUS thalamotomy. Studies suggest that this novel treatment shows potential to reduce ET, and appears to have positive effects on the quality of life of patients with ET. Although not observed, monitoring for serious adverse events, such as intracranial hemorrhage and permanent neurologic impairment, is advised.

Most of the studies were uncontrolled, suggesting the need for blinded and randomized trials to assess the effectiveness of the treatment, the stability of benefits, and the long-term side effects. Additionally, none of the studies used sham procedures to control the placebo effect. The latter appears in the literature as a controversial issue. ${ }^{12}$ Though some argue that the placebo effect should be controlled in surgeries, Albin argues that in order to minimize risk for research subjects, sham surgery controls should not be the default method of constructing human clinical trials involving surgical interventions. There should be an absence of useful objective measures addressing the study outcomes to justify sham surgery controls. ${ }^{13}$

Currently, Elias et al. are conducting a randomized controlled trial of unilateral stereotactic MRIgFUS thalamotomy including seventy-two patients with severe, medication-refractory ET across seven international centers. ${ }^{14}$ This trial, in which Elias et al. randomize patients to receive either sham procedure or MRIgFUS thalamotomy, may shed light on these issues and inform future trials. Qualitative studies exploring the lived experiences of patients with ET who underwent MRIgFUS thalamotomy would provide in-depth understanding of the effects of this treatment in their life.

\section{References}

1. Louis ED, Ferreira JJ. How common is the most common adult movement disorder? Update on the worldwide prevalence of essential tremor. Mov Disorders 2010;25(5):534-41.

2. Dobrakowski PP, Machowska-Majchrzak AK, Labuz-Roszak B, Majchrzak KG, Kluczewska E, Pierzchala KB. MR-guided focused ultrasound: a new generation treatment of Parkinson's disease, essential tremor and neuropathic pain. Interv Neuroradiol 2014; 20(3):275-82.

3. Bain PG, Findley LJ, Thompson PD, Gresty MA, Rothwell JC, Harding AE, et al. A study of hereditary essential tremor. Brain $1994 ; 117(4): 805-24$.

4. LeDoux MS. Non-parkinson movement disorders: five new things. Neurol Clin Pract 2013;3(1):22-9.

5. Elias WJ, Huss D, Voss T, Loomba J, Khaled M, Zadicario E, et al. A pilot study of focused ultrasound thalamotomy for essential tremor. N Engl J Med 2013;369(7):640-8.

6. Wintermark M, Druzgal J, Huss DS, Khaled MA, Monteith S, Raghavan $\mathrm{P}$, et al. Imaging findings in $\mathrm{mr}$ imaging-guided focused ultrasound treatment for patients with essential tremor. Am J Neuroradiol 2014;39(5):891-6.

7. Wintermark M, Huss DS, Shah BB, Tustison N, Druzgal TJ, Kassell $\mathrm{N}$, et al. Thalamic connectivity in patients with essential tremor treated with MR imaging-guided focused ultrasound: in vivo fiber tracking by using diffusion-tensor MR imaging. Radiology 2014; 272(1):202-9.

8. Mandl RCW, Schnack HG, Zwiers MP, van der Schaaf A, Kahn RS, Hulshoff Pol HE. Functional diffusion tensor imaging: measuring task-related fractional anisotropy changes in the human brain along white matter tracts. PLoS One 2008;3(11): e3631.

9. Lipsman N, Schwartz ML, Huang Y, Lee L, Sankar T, Chapman M, et al. MR-guided focused ultrasound thalamotomy for essential tremor: a proof-of-concept study. Lancet Neurol 2013;12(5):4628.

10. Jung HH, Chang WS, Rachmilevitch I, Tlusty T, Zadicario E, Chang JW. Different magnetic resonance imaging patterns after transcranial magnetic resonance-guided focused ultrasound of the ventral intermediate nucleus of the thalamus and anterior limb of the internal capsule in patients with essential tremor or obsessive-compulsive disorder. J Neurosurg 2015;122(1):162-8.

11. Chang JW, Min B-K, Kim B-S, Chang WS, Lee Y-H. Neurophysiologic correlates of sonication treatment in patients with essential tremor. Ultrasound Med Biol 2015 41(1):124-31.

12. Wolf BR, Buckwalter JA. Randomized surgical trials and "sham" surgery: relevance to modern orthopaedics and minimally invasive surgery. Iowa Orthop J 2006;26:107-11.

13. Albin R. Sham surgery controls: intracerebral grafting of fetal tissue for Parkinson's disease and proposed criteria for use of sham surgery controls. J Med Ethics 2002;28(5): 322-5. 